\title{
Health-Related Quality of Life With \\ Carboplatin-Paclitaxel or nab-Paclitaxel With or Without Pembrolizumab in Patients With Metastatic Squamous Non-Small-Cell Lung Cancer
}

Julien Mazieres, MD, PhD; Dariusz Kowalski, MD, PhD²; Alexander Luft, MD, PhD³; David Vicente, MD; Ali Tafreshi, MD; Mahmut Gümüş, MD; Konstantin Laktionov, MD7 Barbara Hermes, MD; Irfan Cicin, MD ${ }^{9}$; Jerónimo Rodríguez-Cid, MD ${ }^{10}$; Jonathan Wilson, MD ${ }^{11}$; Terufumi Kato, MD ${ }^{12}$; Rodryg Ramlau, MD, PhD ${ }^{13}$; Silvia Novello, MD, PhD ${ }^{14}$; Sreekanth Reddy, MD ${ }^{15}$; Hans-Georg Kopp, MD ${ }^{16}$; Bilal Piperdi, MD ${ }^{17}$; Xiaodong Li, PhD ${ }^{17}$; Thomas Burke, PhD ${ }^{17}$; and Luis Paz-Ares, MD, PhD ${ }^{18}$

PURPOSE In the phase 3 KEYNOTE-407 study, the addition of pembrolizumab to carboplatin-paclitaxel/ nab-paclitaxel significantly improved overall survival, progression-free survival, and objective response rate in patients with previously untreated metastatic squamous non-small-cell lung cancer (NSCLC), with little impact on severe toxicity. We present patient-reported outcomes (PROs) from KEYNOTE-407.

METHODS Patients were randomly assigned to receive 4 cycles of pembrolizumab $200 \mathrm{mg}$ or placebo once every 3 weeks plus carboplatin plus paclitaxel or nab-paclitaxel, followed by pembrolizumab or placebo for an additional 31 cycles. Health-related quality of life (HRQoL) was evaluated using the European Organisation for Research and Treatment of Cancer Treatment of Cancer Quality of Life Questionnaire-Core 30 (QLQ-C30) and Quality of Life Questionnaire-Lung Cancer Module 13 (QLQ-LC13). Key PRO endpoints were change from baseline to weeks 9 and 18 (during and after platinum therapy) in the QLQ-C30 global health status/quality of life (GHS/QoL) score and time to deterioration in the composite endpoint of cough, chest pain, or dyspnea from the QLQ-C30 and QLQ-LC13. Two-sided, nominal $P$ values are provided.

RESULTS A total of 554 and 553 patients completed $\geq 1$ QLQ-C30 or $\geq 1$ QLQ-LC13 assessment, respectively. GHS/QoL score improved for the pembrolizumab-combination group (least squares [LS] mean [95\% Cl] change from baseline: week 9, 1.8 [-0.9 to 4.4]; week 18, 4.3 [1.7 to 6.9]) and deteriorated in the placebo-combination group (week $9,-1.8$ [ -4.4 to 0.7]; week 18, -0.57 [ -3.3 to 2.2]). Between-group differences were improved for the pembrolizumab-combination group (difference in LS mean scores: week 9, 3.6 [95\% Cl, 0.3 to 6.9], nominal $P=.0337$; week 18, 4.9 [1.4 to 8.3], nominal $P=.0060$ ). Median time to deterioration in cough, chest pain, or dyspnea was not reached in either group (hazard ratio, $0.79 ; 95 \% \mathrm{Cl}, 0.58$ to 1.06 ]; nominal $P=.125$ ).

ASSOCIATED CONTENT

Protocol

Author affiliations and support information (if applicable) appear at the end of this article.

Accepted on October 1,2019 and published at jco.org on November 21, 2019: DOI https://doi. org/10.1200/JC0.19. 01348

Clinical trial information: NCT02775435.

CONCLUSION Addition of pembrolizumab to chemotherapy maintained or improved HRQoL measurements relative to baseline and improved HRQoL versus chemotherapy alone at weeks 9 and 18. These results support use of pembrolizumab plus chemotherapy as first-line therapy for metastatic squamous NSCLC.

J Clin Oncol 37. @ 2019 by American Society of Clinical Oncology

\section{INTRODUCTION}

For patients with metastatic squamous non-small-cell lung cancer (NSCLC), first-line therapy has typically comprised platinum-taxane chemotherapy. ${ }^{1}$ KEYNOTE407 (ClinicalTrials.gov identifier: NCT02775435) is a randomized phase 3 study of pembrolizumab plus carboplatin-paclitaxel/nab-paclitaxel versus placebo plus carboplatin-paclitaxel/nab-paclitaxel among patients with metastatic squamous NSCLC. Addition of pembrolizumab to carboplatin-paclitaxel/nab-paclitaxel improved overall survival (OS; hazard ratio [HR], 0.64;
95\% Cl, 0.49 to $0.85 ; P<.001$ ) and progression-free survival (PFS; HR, 0.56; $95 \% \mathrm{Cl}, 0.45$ to $0.70 ; P<$ .001) regardless of programmed death-ligand 1 (PDL1) expression. ${ }^{2}$ Incidence and severity of adverse events (AEs) were similar between groups, although the rate of discontinuation due to $A E s$ was higher in the pembrolizumab-combination group versus the placebocombination group.

Disease-related symptoms associated with advanced NSCLC are associated with poor health-related quality of life (HRQoL). ${ }^{3,4}$ Two phase 3 clinical studies of 
pembrolizumab as first-line NSCLC treatment have evaluated changes from baseline in HRQoL and time to deterioration in lung cancer symptoms as prespecified exploratory endpoints. In KEYNOTE-024 (ClinicalTrials.gov identifier: NCT02142738), pembrolizumab monotherapy improved OS and PFS versus platinum-based chemotherapy among patients with previously untreated metastatic NSCLC (any histology; no sensitizing EGFR/ALK alterations) with PD-L1 tumor proportion score (TPS) $\geq 50 \% .{ }^{5}$ Patients who received pembrolizumab also had better global health status/quality of life (GHS/QoL) scores and prolonged time to deterioration in lung cancer symptoms versus those who received chemotherapy. ${ }^{6}$ In KEYNOTE-189 (ClinicalTrials.gov identifier: NCT03950674), pembrolizumab plus pemetrexed-platinum improved OS and PFS and maintained HRQoL, with improved HRQoL over placebo plus pemetrexed-platinum after completion of platinum therapy among patients with metastatic nonsquamous NSCLC without sensitizing EGFR/ALK alterations.

We report patient-reported outcomes (PROs) from KEYNOTE-407, which were evaluated as prespecified exploratory endpoints. These analyses evaluated changes from baseline in GHS/QoL and time to deterioration in lung cancer symptoms.

\section{METHODS}

\section{Study Design and Participants}

KEYNOTE-407 study design and inclusion/exclusion criteria are published elsewhere. ${ }^{2}$ Briefly, eligible patients were $\geq 18$ years of age with histologically/cytologically confirmed stage IV squamous NSCLC without prior systemic treatment of metastatic NSCLC. The study protocol was approved by an independent institutional review board/ ethics review committee at each site.

Patients were randomly assigned $1: 1$ to receive four 3-week cycles of either pembrolizumab $200 \mathrm{mg}$ or saline placebo (day 1 of each cycle) plus carboplatin area under the curve $6 \mathrm{mg} / \mathrm{mL} / \mathrm{min}$ (day 1) and paclitaxel $200 \mathrm{mg} / \mathrm{m}^{2}$ (day 1 ) or nab-paclitaxel $100 \mathrm{mg} / \mathrm{m}^{2}$ (days $1,8,15$ ) at the investigator's discretion. Patients received pembrolizumab $200 \mathrm{mg}$ or placebo once every 3 weeks for up to 35 total cycles (approximately 2 years of treatment in total). Randomization was stratified by chemotherapy regimen (paclitaxel/nab-paclitaxel), PD-L1 expression (TPS $\geq 1 \% /$ TPS $<1 \%$; patients without an evaluable TPS were included in the TPS $<1 \%$ group), and region (East Asia/non-East Asia). Treatment was discontinued on disease progression by local radiologic review per immune-related Response Evaluation Criteria in Solid Tumors (irRECIST), unacceptable toxicity, intercurrent illness preventing further treatment, or patient/investigator decision. Patients with disease progression per RECIST version 1.1 , verified by blinded independent central review (BICR), had their treatment assignment unblinded, and those initially in the placebo-combination group could cross over to receive pembrolizumab monotherapy for up to 35 cycles. Patients in the pembrolizumabcombination group who had documented disease progression but were benefiting clinically could receive openlabel pembrolizumab monotherapy for up to 35 total administrations.

\section{Endpoints}

The primary endpoints were OS and PFS per RECIST version 1.1 by BICR. Secondary endpoints included safety and objective response rate per RECIST version 1.1 by BICR. PROs were evaluated as prespecified exploratory endpoints. HRQoL changes from baseline were evaluated using the European Organisation for Research and Treatment of Cancer (EORTC) Quality of Life QuestionnaireCore 30 (QLQ-C30), ${ }^{8}$ Quality of Life Questionnaire-Lung Cancer Module 13 (QLQ-LC13), ${ }^{9}$ and EuroQoL (EQ)-5D-3L. The EQ-5D-3L was collected to generate utility values for health states required for cost-effectiveness modeling. ${ }^{10}$ Key PRO endpoints were mean score change from baseline to weeks 9 and 18 evaluated by the EORTC QLQ-C30 GHS/QoL and time to deterioration in the composite endpoint of cough (LC13, question 1), chest pain (LC13, question 10), or dyspnea (C30, question 8). Supportive PRO endpoints included mean score changes from baseline in EORTC QLQ-C30 and EORTC QLQ-LC13 subscales, and number and proportion of patients who were "improved," "stable," or "deteriorated" from baseline at weeks 9 and 18. These time points were selected to represent times during (week 9) and after (week 18) chemotherapy treatment and minimize data loss due to disease progression/death. Patients' postbaseline PRO scores were classified as improved, stable, or deteriorated according to $a \geq 10$-point change for each instrument/ scale. A score change of $\geq 10$ points from baseline was used to classify the change as improved or deteriorated; this magnitude is generally accepted by patients as being clinically meaningful. ${ }^{11}$ Effect of disease progression and treatment on mean change from baseline to weeks 9 and 18 in QLQ-C30 GHS/QoL score was evaluated as a supportive analysis.

\section{HRQoL Assessments}

Questionnaires were administered by trained study-site personnel and completed using a tablet computer by each patient before drug administration, AE evaluation, and disease status notification at cycles 1-7 and every third cycle thereafter up to 48 weeks while on treatment and at treatment discontinuation and 30-day safety follow-up visits. Instruments were administered in the following order: EQ-5D-3L, EORTC QLQ-C30, and EORTC QLQ-LC13.

\section{Statistical Analyses}

Planned enrollment was 560 patients. Assuming 361 deaths, this provided approximately $85 \%$ power to detect an 
HR of 0.7 with an alpha of .01 (1 sided). Power calculations were not performed for PRO analyses; $P$ values for all PRO analyses were 2 sided and nominal. There was no adjustment for multiplicity.

PROs were analyzed in the full-analysis population (all randomly assigned patients who received $\geq 1$ dose of study treatment and completed $\geq 1$ PRO assessment). Patients who completed $\geq 1$ item on a PRO instrument were considered to have completed $\geq 1$ PRO assessment. The completion rate was the percentage of patients who completed $\geq 1$ PRO assessment in the fullanalysis population at each time point. Compliance with
PRO assessment was defined as the proportion of patients who completed $\geq 1$ item among those who were expected to complete the questionnaires (ie, those who were alive, had not discontinued, had translation available, and had a scheduled visit).

Evaluation of mean change from baseline to weeks 9 and 18 in the PRO instrument score was based on a constrained longitudinal data analysis model (Data Supplement). The response variable was the PRO score, and the covariates were treatment by study visit and randomization stratification factors. Between-group comparisons were reported as differences in the least

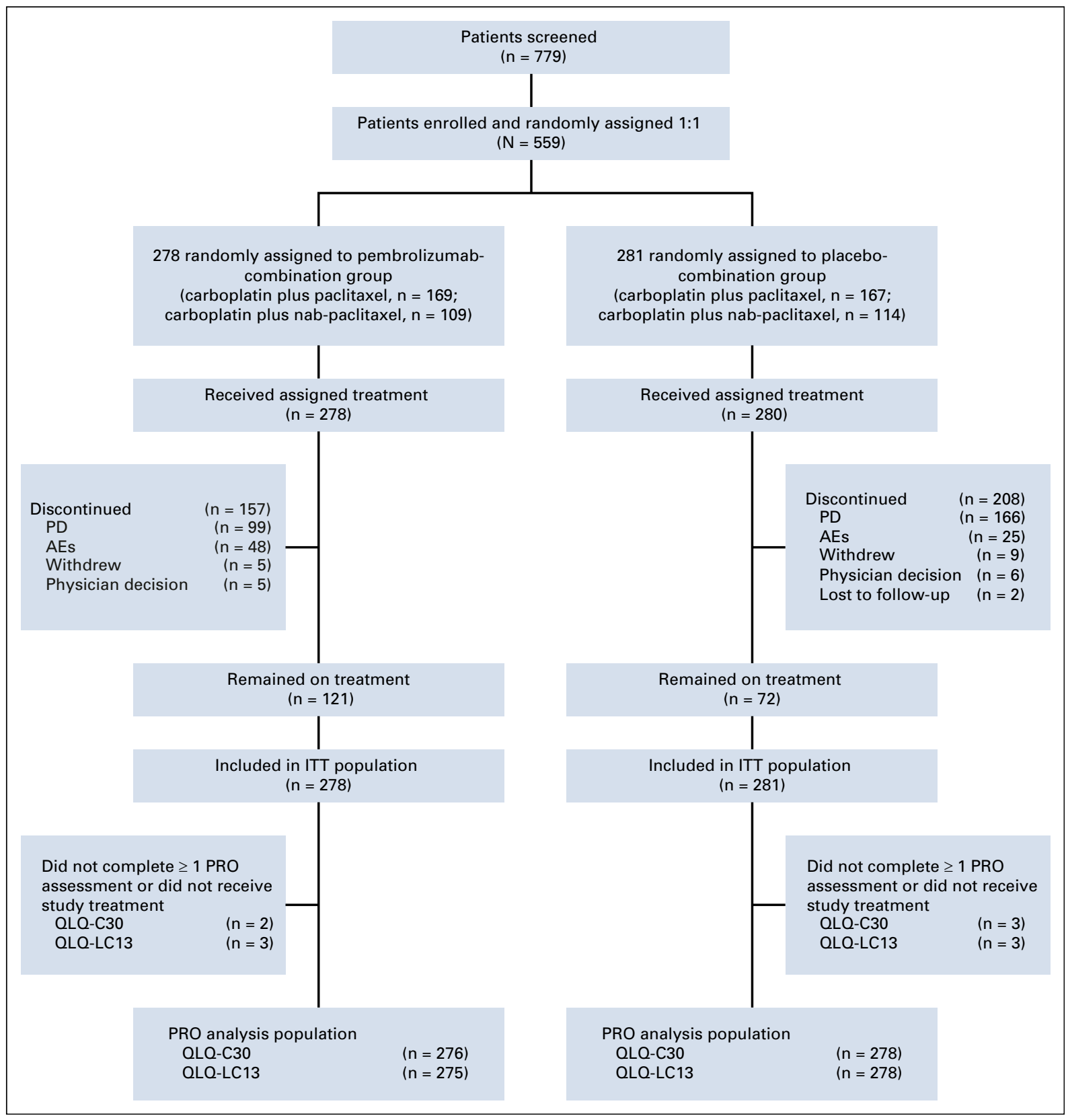

FIG 1. CONSORT diagram. Disposition of patients in the study. AE, adverse event; ITT, intent to treat; PD, progressive disease; PRO, patient-reported outcome; QLC-C30, Quality of Life Questionnaire-Core 30; QLC-LC, Quality of Life Questionnaire-Lung Cancer Module 13. 
TABLE 1. Completion and Compliance Rates for PRO Instruments

\begin{tabular}{|c|c|c|}
\hline Patients & Pembrolizumab-Combination & Placebo-Combination \\
\hline \multicolumn{3}{|l|}{ QLQ-C30 } \\
\hline No. ${ }^{*}$ & 276 & 278 \\
\hline Baseline & 254 (92.0) & $264(95.0)$ \\
\hline \multicolumn{3}{|l|}{ Week 3} \\
\hline Completion & 228 (82.6) & 237 (85.3) \\
\hline Compliance & $228 / 265$ (86.0) & 237/266 (89.1) \\
\hline \multicolumn{3}{|l|}{ Week 6} \\
\hline Completion & 226 (81.9) & 204 (73.4) \\
\hline Compliance & 226/253 (89.3) & 204/251 (81.3) \\
\hline \multicolumn{3}{|l|}{ Week 9} \\
\hline Completion & $187(67.8)$ & 199 (71.6) \\
\hline Compliance & 187/233 (80.3) & 199/225 (88.4) \\
\hline \multicolumn{3}{|l|}{ Week 12} \\
\hline Completion & 194 (70.3) & 177 (63.7) \\
\hline Compliance & 194/227 (85.5) & $177 / 224(79.0)$ \\
\hline \multicolumn{3}{|l|}{ Week 15} \\
\hline Completion & $191(69.2)$ & $165(59.4)$ \\
\hline Compliance & $191 / 224(85.3)$ & 165/201 (82.1) \\
\hline \multicolumn{3}{|l|}{ Week 18} \\
\hline Completion & $191(69.2)$ & $162(58.3)$ \\
\hline Compliance & 191/217 (88.0) & 162/187 (86.6) \\
\hline \multicolumn{3}{|l|}{ QLQ-LC13 } \\
\hline No. ${ }^{*}$ & 275 & 278 \\
\hline Baseline & 252 (91.6) & 263 (94.6) \\
\hline \multicolumn{3}{|l|}{ Week 3} \\
\hline Completion & 227 (82.5) & 237 (85.3) \\
\hline Compliance & 227/265 (85.7) & 237/266 (89.1) \\
\hline \multicolumn{3}{|l|}{ Week 6} \\
\hline Completion & 226 (82.2) & 204 (73.4) \\
\hline Compliance & 226/253 (89.3) & 204/251 (81.3) \\
\hline \multicolumn{3}{|l|}{ Week 9} \\
\hline Completion & $187(68.0)$ & 197 (70.9) \\
\hline Compliance & 187/233 (80.3) & 197/225 (87.6) \\
\hline \multicolumn{3}{|l|}{ Week 12} \\
\hline Completion & $192(69.8)$ & 175 (62.9) \\
\hline Compliance & $192 / 227(84.6)$ & $175 / 224(78.1)$ \\
\hline \multicolumn{3}{|l|}{ Week 15} \\
\hline Completion & 191 (69.5) & $164(59.0)$ \\
\hline Compliance & $191 / 224(85.3)$ & 164/201 (81.6) \\
\hline \multicolumn{3}{|l|}{ Week 18} \\
\hline Completion & $191(69.5)$ & $162(58.3)$ \\
\hline Compliance & 191/217 (88.0) & $162 / 187(86.6)$ \\
\hline
\end{tabular}

NOTE. Data presented as No. (\%) unless otherwise indicated. Compliance rate was defined as the percentage of patients who completed the PRO questionnaire among those who were expected to complete the instrument at each time point (eg, those who had not discontinued study treatment). Completion rate was defined as the proportion of patients in the analysis population at each time point who completed $\geq 1$ assessment.

Abbreviations: PRO, patient-reported outcome; QLQ-C30, Quality of Life Questionnaire-Core 30; QLQ-LC13, Quality of Life Questionnaire-Lung Cancer Module 13.

*The PRO analysis population included patients who completed $\geq 1$ treatment dose and $\geq 1$ PRO assessment squares (LS) mean change from baseline with the 95\% $\mathrm{Cl}$ and nominal $P$ value. Time to deterioration in the composite of cough, chest pain, or dyspnea was defined as time to first onset of $\geq 10$-point increase from baseline with confirmation by a second adjacent $\geq 10$ point increase in any of the 3 symptoms under the right-censoring rule. The nonparametric Kaplan-Meier method was used to estimate the deterioration curve in each group; a stratified Cox model with Efron's method of tie handling was used to assess between-group differences. Proportions of patients with improved, stable, and deteriorated scores at weeks 9 and 18 were summarized based on multiple imputation for missing data with missing at random assumption and then synthesized based on Rubin's rule. QLQ-C30/QLQ-LC13 scores were standardized to a scale ranging from 0-100 by linear transformation. For GHS/QoL and functional scales, higher scores indicate a higher (better) level of function, whereas for the symptom scales, higher scores indicate a higher (worse) severity of symptoms.

\section{RESULTS}

\section{Patients}

Between August 19, 2016, and December 28, 2017, 559 patients were randomly assigned to pembrolizumab plus chemotherapy ( $n=278$ ) or placebo plus chemotherapy ( $n=281$; Fig 1 ). As of the data cutoff (April 3, 2018), median follow-up was 7.8 months (range, 0.1-19.1 months). As reported previously, baseline characteristics were similar between groups. ${ }^{2}$

With the exception of 1 patient in the placebo-combination group, all enrolled patients received $\geq 1$ dose of assigned study treatment. Among these, the $\mathrm{PRO}$ analysis population comprised 554 patients who completed $\geq 1$ QLQ-C30 assessment and 553 patients who completed $\geq 1$ QLQLC13 assessment. Compliance rates were similar between groups at baseline and weeks 9 and 18, and remained high at both time points (Table 1). Completion rates for the QLQC30 and QLQ-LC13 were high at baseline (>90\%) for both groups (Table 1). At week 9 , completion rates were $67.8 \%$ and $71.6 \%$, respectively, for the QLQ-C30 and $68.0 \%$ and $70.9 \%$, respectively, for the QLQ-LC13. At week 18, completion rates were $69.2 \%$ and $58.3 \%$ for the QLQ-C30, and $69.5 \%$ and $58.3 \%$ for the QLQ-LC13 (Table 1). The decline in completion rates at week 18 was associated with an increase in the number of patients who discontinued because of an $\mathrm{AE}$, physician decision, disease progression, study withdrawal, or death, or who had no scheduled visit. Between-group differences at week 18 were mainly attributed to an increase in the number of patients in the placebo-combination group who were missing an $\mathrm{HRQOL}$ instrument due to disease progression versus the pembrolizumab-combination group (QLQ-C30: 4.0\%, 11/ 276 v 17.3\%, 48/278; QLQ-LC13: 3.6\%, 10/275 v 17.3\%, 48/278). 


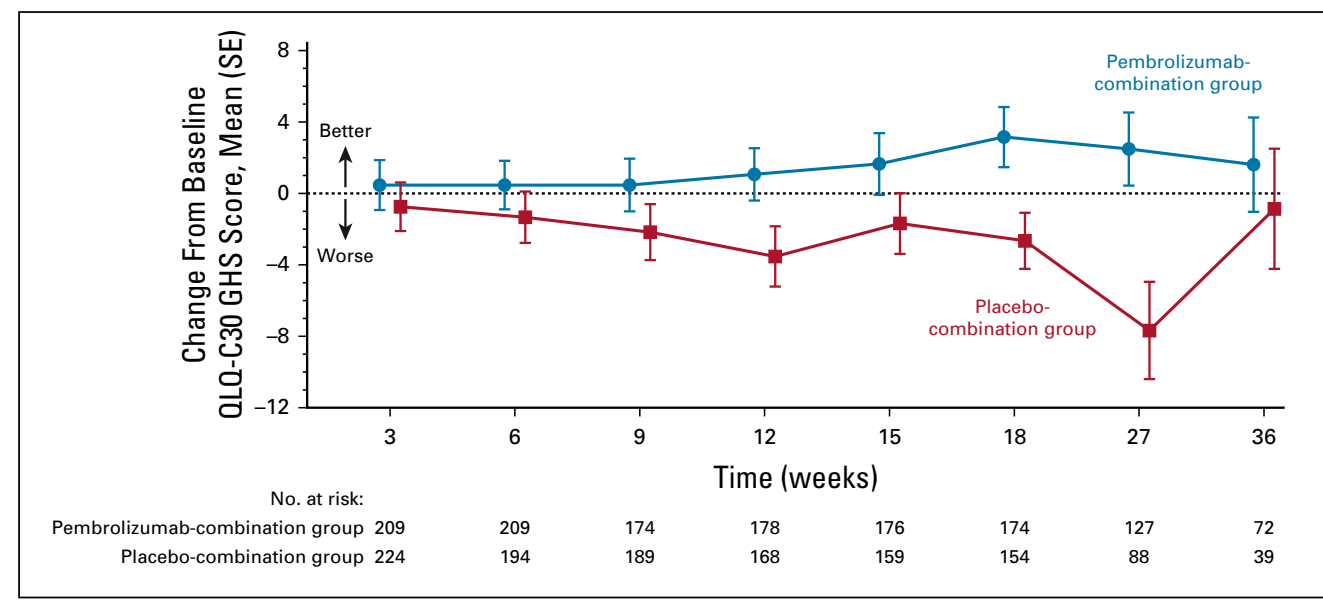

FIG 2. Mean change from baseline in Quality of Life Questionnaire-Core 30 (QLQ-C30) global health status (GHS) scores by visit.

\section{Key PRO Endpoints}

Baseline mean (SD) QLQ-C30 GHS/QoL scores were similar between the pembrolizumab-combination group (63.9 [20.4]) and placebo-combination groups (62.7 [21.3]). For the pembrolizumab-combination group, mean QLQ-C30 GHS/QoL scores were above baseline at all time points, with the largest improvements starting at week 18. For the placebo-combination group, mean QLQ-C30 GHS/QoL scores were lower versus baseline at all assessments through week 36 (Fig 2).
At week 9, LS mean (95\% Cl) QLQ-C30 GHS/QoL scores were stable in the pembrolizumab-combination group (increase of 1.8 points [ -0.9 to 4.4$]$ ) and the placebocombination group (decrease by 1.8 points [ -4.4 to 0.7$]$ ). The LS mean difference in score between groups at week 9 was 3.6 points $(95 \% \mathrm{Cl}, 0.3$ to $6.9 ; P=.0337)$. At week 18 , the LS mean (95\% Cl) QLQ-C30 GHS/QoL score was improved from baseline in the pembrolizumab-combination group (increase of 4.3 points [1.7 to 6.9$]$ ), whereas it was stable in the placebo-combination group (decrease by 0.6

TABLE 2. Mean Changes From Baseline in QLQ-C30 GHS/QoL Score

\begin{tabular}{|c|c|c|c|}
\hline Time Point & $\begin{array}{l}\text { Pembrolizumab-Combination } \\
\qquad(\mathrm{n}=276)\end{array}$ & $\begin{array}{l}\text { Placebo-Combination } \\
\quad(\mathrm{n}=278)\end{array}$ & $P$ \\
\hline \multicolumn{4}{|l|}{ Baseline } \\
\hline Completed questionnaire, No. & 254 & 264 & \\
\hline Mean score (SD) & $63.9(20.4)$ & $62.7(21.3)$ & \\
\hline \multicolumn{4}{|l|}{ Week 9} \\
\hline Completed questionnaire, No. & 187 & 199 & \\
\hline Mean score (SD) & $66.0(18.5)$ & $62.1(19.6)$ & \\
\hline \multicolumn{4}{|l|}{ Change from baseline* } \\
\hline Included in analysis, No. & 276 & 278 & \\
\hline LS mean score $(95 \% \mathrm{Cl})$ & $1.8(-0.9$ to 4.4$)$ & $-1.8(-4.4$ to 0.7$)$ & \\
\hline Difference in LS mean $(95 \% \mathrm{Cl})$ & 3.6( & & $P=.0337 \dagger$ \\
\hline \multicolumn{4}{|l|}{ Week 18} \\
\hline Completed questionnaire, No. & 191 & 162 & \\
\hline Mean score (SD) & $68.9(19.3)$ & $65.2(17.1)$ & \\
\hline \multicolumn{4}{|l|}{ Change from baseline* } \\
\hline Included in analysis, No. & 276 & 278 & \\
\hline LS mean score $(95 \% \mathrm{Cl})$ & 4.3 (1.7 to 6.9$)$ & $-0.6(-3.3$ to 2.2$)$ & \\
\hline Difference in LS mean $(95 \% \mathrm{CI})$ & 4.9( & & $P=.0060 \dagger$ \\
\hline
\end{tabular}

Abbreviations: GHS/QoL, global health status/quality of life; LS, least squares; QLQ-C30, Quality of Life Questionnaire-Core 30; SD, standard deviation.

*Based on a constrained longitudinal data analysis model with QLQ-C30 GHS/QoL scores as the response variable and treatment by study visit interaction and randomization stratification factors as covariates.

$\dagger P$ values are 2 sided and nominal. 


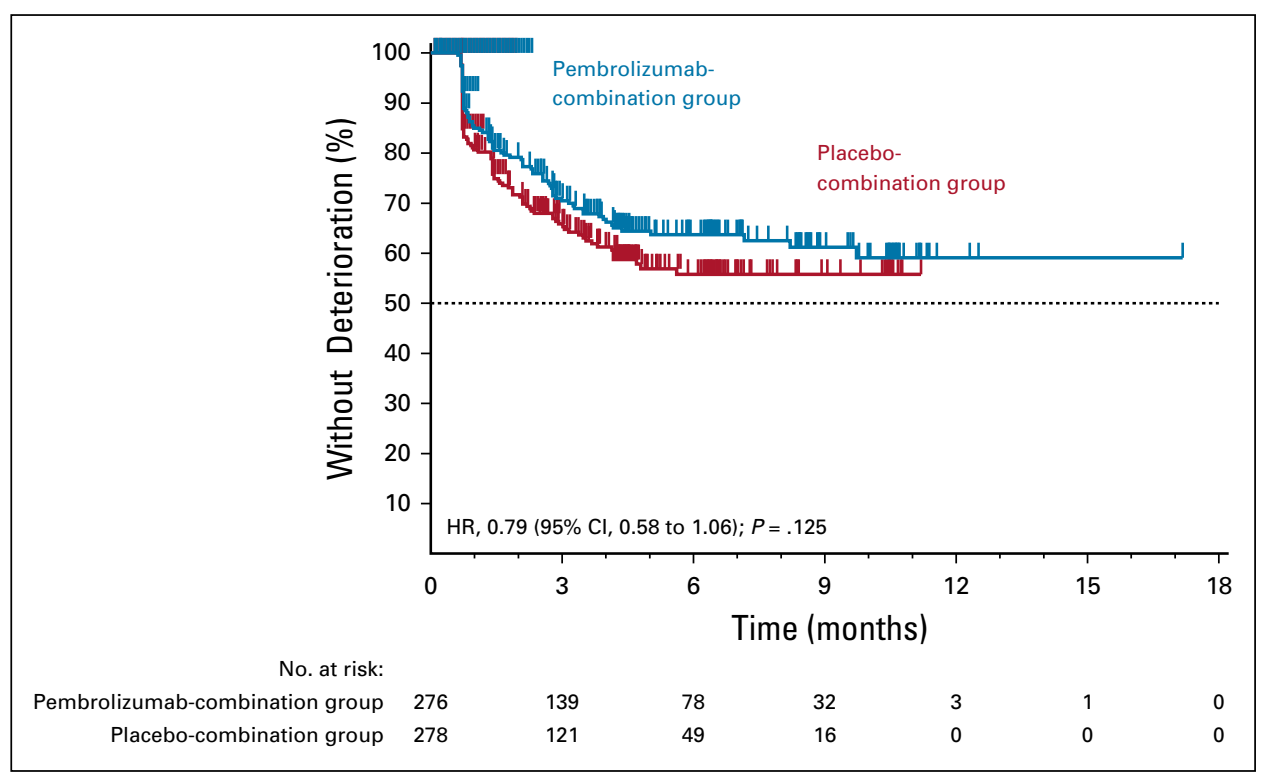

FIG 3. Time to deterioration in the composite endpoint of cough (Quality of Life Questionnaire-Lung Cancer Module 13 [QLQ-LC13], question 1), chest pain (QLQLC13, question 10), or dyspnea (Quality of Life Questionnaire-Core 30, question 8). $\mathrm{HR}$, hazard ratio.

points [ -3.3 to 2.2]). The LS mean difference in score between groups at week 18 was 4.9 points $(95 \% \mathrm{Cl}, 1.4$ to 8.3; $P=.0060$; Table 2).

Eighty-one patients $(29.3 \%)$ in the pembrolizumabcombination group and 94 patients (33.8\%) in the placebo-combination group experienced deterioration in the composite endpoint of cough, chest pain, or dyspnea. Median time to deterioration for these symptoms was not reached in either group ( $\mathrm{HR}, 0.79 ; 95 \% \mathrm{Cl}, 0.58$ to 1.06 ; $P=.125$; Fig 3).

\section{Supportive PRO Endpoints}

The EORTC QLQ-C30 instrument includes 5 functional domains (physical, role, emotional, cognitive, and social) that are used to assess HRQoL in patients with cancer. Change from baseline scores was numerically superior for the pembrolizumab-combination group versus the placebo-combination group across all functional scales at weeks 9 and 18 (Figs $4 \mathrm{~A}$ and 4B). For the pembrolizumabcombination group, there were minimal changes from baseline in physical, cognitive, role, and social function scales at weeks 9 and 18, and improvements occurred in emotional functioning score at both time points. In the placebo-combination group at weeks 9 and 18, scores declined from baseline for physical and role functioning, there were minimal changes for cognitive and social functioning, and improvements occurred for emotional functioning (Figs 4A and 4B).

The EORTC QLQ-C30 instrument also includes 3 symptom scales (fatigue, pain, and nausea/vomiting) and 6 single items assessing other symptoms common among patients with cancer. Both treatment groups were improved (lower scores) in most of the scales at week 9 (during taxane therapy), with further improvements at week 18 (after taxane therapy). There were increases in symptoms of fatigue, nausea/vomiting, and diarrhea at week 9 for both treatment groups, and an increase in diarrhea symptoms at week 18 for the pembrolizumabcombination group. At both time points, the pembrolizumabcombination group was numerically superior in fatigue, pain, dyspnea, and insomnia, whereas the placebo-combination group was numerically superior in the scales of nausea/ vomiting, appetite loss, constipation, and diarrhea (Figs 4C and 4D).

Fewer patients in the pembrolizumab-combination group reported a deterioration in GHS/QoL status versus the placebo-combination group (week 9: 26.1\% v 29.5\%; week 18: $22.8 \% \vee 31.3 \%)$. More patients in the pembrolizumab-combination group reported an improved GHS/QoL status versus the placebo-combination group (week 9: 30.4\% v 24.5\%; week 18: 36.2\% $v$ 27.7\%; Fig 5). There were fewer patients with a deterioration in physical functioning in the pembrolizumabcombination group compared with the placebo-combination group (week 9: 29.7\% v35.3\%; week 18: $26.1 \%$ v. $37.4 \%$ ). At weeks 9 and 18, the percentage of patients with improved/ stable scores across all functional scales was greater in the pembrolizumab-combination group versus the placebocombination group. There were limited differences in distribution of improved, stable, and deteriorated scores on the symptom scales at week 9 between the pembrolizumabcombination and placebo-combination groups. More patients in the pembrolizumab-combination group versus patients in the placebo-combination group had improved symptom scale scores for fatigue (week 9, 37.0 v 33.1; week $18,46.0 \% \vee 37.1 \%$ ), nausea and vomiting (week 9 , $14.9 \vee 12.2$; week 18, 16.3\% v 13.3\%), pain (week 9, 39.1 v 36.3; week 18, 40.2\% v 35.3\%), dyspnea (week $9,35.5$ v 27.3; week $18,41.3 \%$ v 28.8\%), and insomnia (week $9,34.1$ v 32.4; week $18,34.4 \%$ vs $31.7 \%$ ). In 


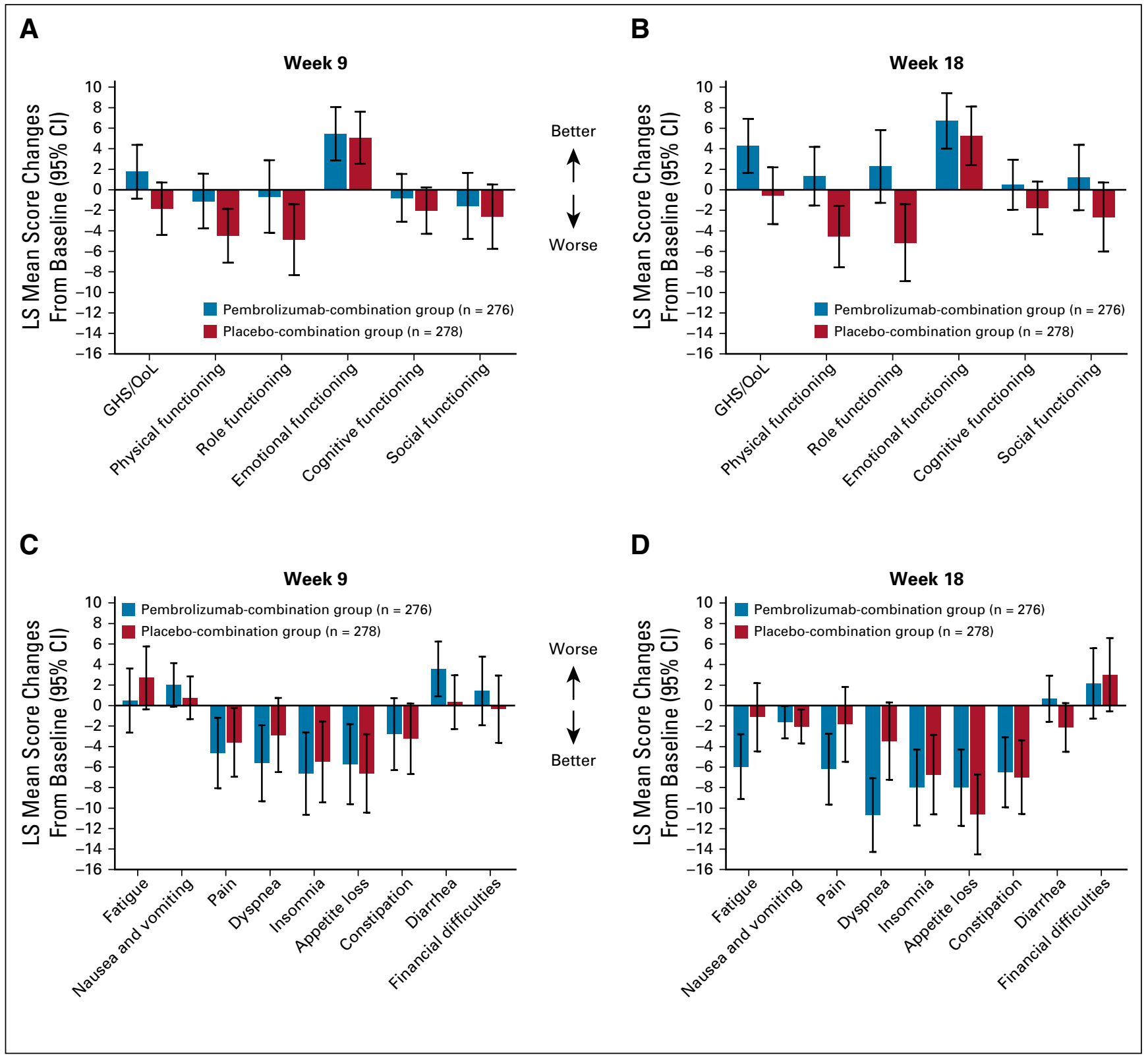

FIG 4. Changes from baseline in Quality of Life Questionnaire-Core 30 (QLQ-C30) global health status/quality of life (GHS/QoL) and functional scale scores at (A) week 9 and (B) week 18, and QLQ-C30 symptom subscale scores at (C) week 9 and (D) week 18. Higher functional scale scores represent better functioning, whereas higher symptom subscale scores indicate more symptoms; higher GHS/QoL represents better health status/QoL.

contrast, the proportion of patients in the pembrolizumabcombination group with deteriorated status was greater for constipation (week 9, 23.2\% v 20.1\%; week 18, 19.6\% v $19.1 \%$ ) and diarrhea (week 9, 24.3\% v 19.4\%; week 18, $18.5 \%$ v 12.6\%; Fig 5).

Analysis of the effect of disease progression on changes from baseline in QLQ-C30 GHS/QoL score showed that among patients with disease progression there were small declines in GHS/QoL in both treatment arms at weeks 9 and 18 (Data Supplement). Among patients without disease progression, GHS/QoL improved in the pembrolizumabcombination arm and declined in the chemotherapy arm at week 9 , and at week 18 was further improved in the pembrolizumab-combination arm, but was little changed from baseline in the chemotherapy arm.

\section{DISCUSSION}

Pembrolizumab plus carboplatin and paclitaxel/nabpaclitaxel maintained or improved HRQoL versus baseline in patients with previously untreated metastatic squamous NSCLC, whereas placebo plus carboplatin and paclitaxel/ nab-paclitaxel led to declines in HRQoL. Baseline QLQC30 GHS/QoL scores were approximately 63 in both treatment groups, which is higher (better) than EORTC 


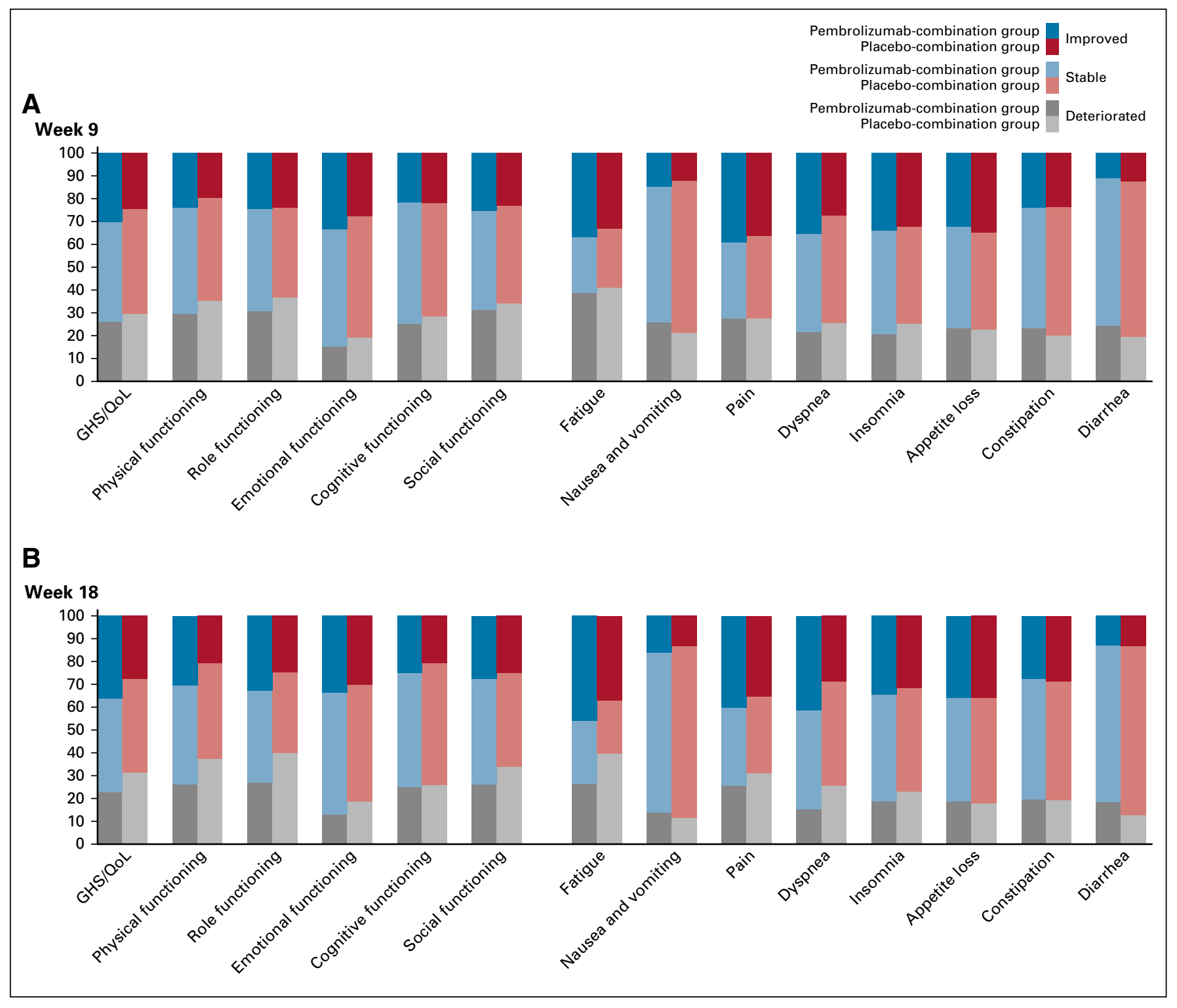

FIG 5. Proportions of patients with improved, stable, and deteriorated (A) Quality of Life Questionnaire-Core 30 global health status/quality of life (GHS/QoL) and functional scale scores, and (B) symptom scale scores at weeks 9 and 18.

GHS/QoL reference values for patients with advanced lung cancer before treatment (mean [SD], 54.7 [23.8]). ${ }^{12}$

Pembrolizumab-combination therapy improved HRQoL versus placebo-combination therapy at week 18 , based on a between-group difference in LS mean scores of 4.9 (95\% Cl, 1.4 to 8.3; $P=.0060$ ). This difference is clinically meaningful, as supported by the GHS/QoL responder analysis: a higher percentage of patients in the pembrolizumab-combination group were classified as improved $(36.2 \% \vee 27.7 \%)$ and fewer were classified as deteriorated $(22.8 \% \vee 31.3 \%)$ at week 18 versus the placebo-combination group. Week 9 between-group differences (ie, during platinum-taxane therapy) favored the pembrolizumab-combination group versus the placebo-combination group, but were smaller in magnitude than those at week 18 (occurring after platinumtaxane therapy). Specifically, more patients in the pembrolizumab-combination group were classified as improved for GHS/QoL and fewer were classified as deteriorated at week 9.

At the data cutoff (median follow-up, 7.8 months), approximately one third of patients had experienced deterioration in the composite endpoint of cough, chest pain, or dyspnea; therefore, median time to deterioration had not been reached in either group. Although the $95 \% \mathrm{Cl}$ for the HR for time to deterioration in these symptoms contained 1 , these results support a trend of a delay in deterioration in the pembrolizumab-combination group ( $\mathrm{HR}, 0.79 ; 95 \% \mathrm{Cl}$, 0.58 to $1.06 ; P=.125)$. These outcomes are clinically meaningful in the context of demographic/clinical 
characteristics of the KEYNOTE-407 study population, ${ }^{2}$ most of whom were current/former smokers, $\geq 65$ years of age, with an Eastern Cooperative Oncology Group performance status of 1 .

HRQoL results favoring the pembrolizumab-combination group at week 18 were supported by trends in the EORTC functional scales, which favored the pembrolizumabcombination group across all scales at weeks 9 and 18 . The largest differences occurred in physical functioning (abilities essential for maintaining independence) and role functioning (abilities for work/leisure). Improvements in physical and role functioning are relevant, given the anticipated continued increase in cancer survivorship ${ }^{13,14}$ treatment regimens may differentially affect patient functioning, and physical and role functional status is likely to affect HRQoL. The symptom scale results include trends favoring each treatment group. The pembrolizumabcombination group had better (lower score) symptom scores for fatigue, pain, dyspnea, and insomnia versus the placebo-combination group. However, these were offset by better (lower scores) for Gl or related symptoms (nausea/ vomiting, diarrhea, constipation, and appetite loss) for the placebo-combination group. These findings are consistent with the incidence of these AEs in the primary assessment of efficacy, with the exception that decreased appetite occurred more frequently in the placebo-combination group. ${ }^{2}$ Potentially, differences in frequency, duration, and/or severity of such events may have contributed to this difference.

PROs from KEYNOTE-407 are consistent with other studies demonstrating a benefit of pembrolizumab on QoL. Pembrolizumab monotherapy improved or maintained HRQoL and delayed deterioration versus platinum-based chemotherapy in patients with stage IV NSCLC in the KEYNOTE024 study. ${ }^{6}$ Similarly, addition of pembrolizumab to pemetrexed-platinum chemotherapy maintained GHS/QoL relative to baseline, with improved GHS/QoL over placebo plus pemetrexed-platinum chemotherapy at week 21 for patients with nonsquamous NSCLC in the KEYNOTE-189 study. ${ }^{15}$ Although cross-trial comparisons are challenging,

\footnotetext{
AFFILIATIONS

${ }^{1}$ Centre Hospitalier Universitaire de Toulouse, Université Paul Sabatier, Toulouse, France

${ }^{2}$ Maria Skłodowska-Curie Institute of Oncology, Warsaw, Poland

${ }^{3}$ Leningrad Regional Clinical Hospital, St Petersburg, Russia

${ }^{4}$ Hospital Universitario Virgen Macarena, Seville, Spain

${ }^{5}$ Wollongong Oncology and University of Wollongong, Wollongong, NSW, Australia

${ }^{6}$ Istanbul Medeniyet University Hospital, Istanbul, Turkey

${ }^{7}$ N.N. Blokhin Russian Cancer Research Center, Moscow, Russia

${ }^{8}$ Universitätsklinikum Tübingen, Tübingen, Germany

${ }^{9}$ Trakya University, Edirne, Turkey

${ }^{10}$ Oncology Center, Medica Sur Hospital, Mexico City, Mexico

${ }^{11}$ Humber River Regional Hospital, Toronto, ON, Canada

${ }^{12}$ Kanagawa Cancer Center, Yokohama, Japan

${ }^{13}$ Poznan University of Medical Sciences, Poznan, Poland
}

the magnitude of improvement in HRQoL in KEYNOTE-407 appeared somewhat smaller than in KEYNOTE-024, but remarkably consistent with KEYNOTE-189. In KEYNOTE407 and KEYNOTE-189, between-group differences in GHS/QoL scores for the pembrolizumab-combination therapies versus placebo-combination therapies were similar at each time point (difference in LS mean scores: 3.6 for week $9 v 3.6$ for week 12; 4.9 for week $18 v 5.3$ for week 21). Moreover, median time to deterioration was not reached in either trial for the pembrolizumab-combination therapies (HR [95\% Cl]: KEYNOTE-407, 0.79 [0.58 to 1.06]; KEYNOTE-189: 0.81 [0.60 to 1.09]). Importantly, in this study, addition of pembrolizumab to chemotherapy improved PROs, even in the context of the toxicity typically associated with platinum-taxane therapy.

The main limitation of this study was the limited follow-up period in assessing PROs. The limited follow-up was based, in part, on the unblinding of the study at the second interim analysis (following the recommendation of the external data safety and monitoring board). Another limitation was the decreasing PRO completion rates with increasing follow-up and the moderate difference in completion rates between groups owing to disease progression in the placebocombination group. In addition, the PRO change scores reported here may not be generalizable to populations with different baseline PRO values. A strength of this study was the double-blind design, which reduced bias in PRO responses relative to an open-label study design.

In conclusion, pembrolizumab plus carboplatin and paclitaxel/nab-paclitaxel maintained or improved HRQoL compared with baseline and improved HRQoL compared with placebo plus carboplatin and paclitaxel/nab-paclitaxel at week 18, as assessed by the EORTC QLQ-C30 and QLQLC13 instruments. These HRQoL findings, along with the improved efficacy (including OS benefit) of pembrolizumab plus carboplatin and paclitaxel/nab-paclitaxel, support its use as a first-line treatment of metastatic squamous NSCLC, regardless of PD-L1 expression.

\footnotetext{
${ }^{14}$ University of Turin, AOU San Luigi, Orbassano, Italy

${ }^{15}$ Northside Hospital Cancer Institute, Atlanta, GA

${ }^{16}$ Robert-Bosch Cancer Center, Klinik Schillerhöhe, Gerlingen, Germany

${ }^{17}$ Merck \& Co., Inc., Kenilworth, NJ

${ }^{18}$ Hospital Universitario 12 de Octubre, CNIO-H120 Lung Cancer Unit, Universidad Complutense and Ciberonc, Madrid, Spain
}

\section{CORRESPONDING AUTHOR}

Julien Mazieres, Thoracic Oncology Department, Hôpital Larrey, CHU Toulouse, Chemin de Pouvourville, 31059 Toulouse, France; e-mail: mazieres.j@chu-toulouse.fr.

\section{PRIOR PRESENTATION}

Presented in part at the European Society for Medical Oncology Congress 2018, Munich, Germany, October 19-23, 2018. 


\section{SUPPORT}

Supported by Merck Sharp \& Dohme, a subsidiary of Merck \& Co., Inc., Kenilworth, NJ

\section{AUTHORS' DISCLOSURES OF POTENTIAL CONFLICTS OF INTEREST} AND DATA AVAILABILITY STATEMENT

Disclosures provided by the authors and data availability statement (if applicable) are available with this article at DOI https://doi.org/10.1200/ JC0.19.01348

\section{AUTHOR CONTRIBUTIONS}

Conception and design: Julien Mazieres, Dariusz Kowalski, David Vicente Mahmut Gümüs, Konstantin Laktionov, Bilal Piperdi, Thomas Burke, Luis Paz-Ares

Financial support: Thomas Burke

Administrative support: Thomas Burke

Provision of study materials or patients: Julien Mazieres, Barbara Hermes, Jerónimo Rodríguez-Cid, Jonathan Wilson, Terufumi Kato, Silvia Novello, Hans-Georg Kopp

Collection and assembly of data: Julien Mazieres, Dariusz Kowalski, Alexander Luft, Ali Tareshi, Mahmut Gümüs, Konstantin Laktionov,
Barbara Hermes, Jerónimo Rodríguez-Cid, Jonathan Wilson, Terufumi Kato, Terufumi Kato, Sreekanth Reddy, Bilal Piperdi

Data analysis and interpretation: Julien Mazieres, Dariusz Kowalski, David Vicente, Mahmut Gümüs, Konstantin Laktionov, Barbara Hermes, Irfan Cicin, Terufumi Kato, Terufumi Kato, Silvi, Novello, Hans-Georg Kopp, Bilal Piperdi, Xiaodong Li, Thomas Burke, Luis Paz-Ares

Manuscript writing: All authors

Final approval of manuscript: All authors

Accountable for all aspects of the work: All authors

\section{ACKNOWLEDGMENT}

We thank the patients and their families and caregivers for participating in this study, along with all investigators and site personnel. Additionally, we acknowledge helpful comments provided on a draft of this article by Jonathan Cheng (Merck \& Co. Inc., Kenilworth, NJ). Medical writing assistance was provided by Charlotte Majerczyk, PhD, of C4

MedSolutions, Yardley, PA, a CHC Group company. This assistance was funded by Merck Sharp \& Dohme Corp., a subsidiary of Merck \& Co., Inc., Kenilworth, NJ.

\section{REFERENCES}

1. National Comprehensive Cancer Network: NCCN clinical practice guidelines in oncology. Non-small cell lung cancer. Version 5.2018. https://www.nccn.org

2. Paz-Ares L, Luft A, Vicente D, et al: Pembrolizumab plus chemotherapy for squamous non-small-cell lung cancer. N Engl J Med 379:2040-2051, 2018

3. Iyer S, Roughley A, Rider A, et al: The symptom burden of non-small cell lung cancer in the USA: A real-world cross-sectional study. Support Care Cancer 22: 181-187, 2014

4. Iyer S, Taylor-Stokes G, Roughley A: Symptom burden and quality of life in advanced non-small cell lung cancer patients in France and Germany. Lung Cancer 81:288-293, 2013

5. Reck M, Rodríguez-Abreu D, Robinson AG, et al: Pembrolizumab versus chemotherapy for PD-L1-positive non-small-cell lung cancer. N Engl J Med 375: 1823-1833, 2016

6. Brahmer JR, Rodríguez-Abreu D, Robinson AG, et al: Health-related quality-of-life results for pembrolizumab versus chemotherapy in advanced, PD-L1positive NSCLC (KEYNOTE-024): A multicentre, international, randomised, open-label phase 3 trial. Lancet Oncol 18:1600-1609, 2017

7. Gandhi L, Rodríguez-Abreu D, Gadgeel S, et al: Pembrolizumab plus chemotherapy in metastatic non-small-cell lung cancer. N Engl J Med 378:2078-2092, 2018

8. Aaronson NK, Ahmedzai S, Bergman B, et al: The European Organization for Research and Treatment of Cancer QLQ-C30: A quality-of-life instrument for use in international clinical trials in oncology. J Natl Cancer Inst 85:365-376, 1993

9. Bergman B, Aaronson NK, Ahmedzai S, et al: The EORTC QLQ-LC13: A modular supplement to the EORTC Core Quality of Life Questionnaire (QLQ-C30) for use in lung cancer clinical trials. Eur J Cancer 30A:635-642, 1994

10. Insinga RP, Vanness DJ, Feliciano JL, et al: Cost-effectiveness of pembrolizumab in combination with chemotherapy versus chemotherapy and pembrolizumab monotherapy in the first-line treatment of squamous non-small-cell lung cancer in the US. Curr Med Res Opin 35:1241-1256, 2019

11. Osoba D, Rodrigues G, Myles J, et al: Interpreting the significance of changes in health-related quality-of-life scores. J Clin Oncol 16:139-144, 1998

12. Scott NW, Fayers PM, Aaronson NK, et al: EORTC QLQ-C30 reference values. https://www.eortc.org/app/uploads/sites/2/2018/02/reference_values_manual2008.pdf

13. Xia W, Yu X, Mao Q, et al: Improvement of survival for non-small cell lung cancer over time. OncoTargets Ther 10:4295-4303, 2017

14. Miller KD, Siegel RL, Lin CC, et al: Cancer treatment and survivorship statistics, 2016. CA Cancer J Clin 66:271-289, 2016

15. Garassino M, Rodríguez-Abreu D, Gadgeel S, et al: Health-related quality of life (HRQoL) in the KEYNOTE-189 study of pembrolizumab (pembro) or placebo (pbo) + pemetrexed (pem) and platinum (plt) for metastatic NSCLC. J Clin Oncol 36:9021, 2018 (suppl; abstr 9021) 
Health-Related Quality of Life With Carboplatin-Paclitaxel or nab-Paclitaxel With or Without Pembrolizumab in Patients With Metastatic Squamous Non-Small-Cell Lung Cancer

The following represents disclosure information provided by authors of this manuscript. All relationships are considered compensated unless otherwise noted. Relationships are self-held unless noted. I = Immediate Family Member, Inst = My Institution. Relationships may not relate to the subject matter of this manuscript. For more information about ASCO's conflict of interest policy, please refer to www.asco.org/rwc or ascopubs.org/jco/site/ifc.

Open Payments is a public database containing information reported by companies about payments made to US-licensed physicians (Open Payments)

\section{Julien Mazieres}

Consulting or Advisory Role: Novartis, Genentech, Pfizer, Bristol-Myers Squibb Eli Lilly/lmClone, MSD, AstraZeneca, Pierre Fabre

Research Funding: Roche (Inst), Bristol-Myers Squibb (Inst), AstraZeneca (Inst) Travel, Accommodations, Expenses: Pfizer, Roche, Bristol-Myers Squibb

Dariusz Kowalski

Consulting or Advisory Role: Bristol-Myers Squibb, Boehringer Ingelheim, Merck Serono, Genentech

\section{David Vicente}

Honoraria: AstraZeneca

Consulting or Advisory Role: Bristol-Myers Squibb, MSD Oncology, Genentech, Pfizer, AstraZeneca, Boehringer Ingelheim

Travel, Accommodations, Expenses: AstraZeneca

Mahmut Gümüş

Consulting or Advisory Role: Roche, Eli Lilly (Inst), Amgen (Inst)

Speakers' Bureau: Roche (Inst), MSD Oncology (Inst)

Travel, Accommodations, Expenses: Pfizer

Barbara Hermes

Research Funding: MSD Oncology (Inst), Novartis (Inst), AstraZeneca (Inst), Bristol-Myers Squibb (Inst), Roche Pharma (Inst), Bayer Health (Inst),

PharmaMar (Inst), Eli Lilly (Inst)

Travel, Accommodations, Expenses: PharmaMar, Eli Lilly

Irfan Cicin

Consulting or Advisory Role: Pfizer (Inst), MSD Oncology (Inst), Roche (Inst), Novartis/Ipsen (Inst), Eli Lilly (Inst), Bristol-Myers Squibb (Inst), Servier (Inst), Abdi Ibrahim (Inst), Nobelpharma (Inst)

Speakers' Bureau: Novartis (Inst), Roche (Inst), Bristol-Myers Squibb (Inst), Pfizer (Inst), Abdi Ibrahim (Inst)

\section{Jerónimo Rodríguez-Cid}

Consulting or Advisory Role: Roche, Bristol-Myers Squibb (Mexico), MSD Oncology, Takeda, Bayer

Speakers' Bureau: MSD Oncology, Bristol-Myers Squibb (Mexico), Roche, Boehringer Ingelheim, Novartis, Bayer, Eli Lilly, AztraZeneca

Research Funding: MSD, Bristol-Myers Squibb (Mexico), Roche, Celltrion, Eli Lilly, BeiGene, AztraZeneca

Travel, Accommodations, Expenses: Roche, MSD Oncology, AztraZeneca, Boehringer Ingelheim

\section{Jonathan Wilson}

Speakers' Bureau: AstraZeneca, Janssen, Pfizer

Research Funding: Eli Lilly (Inst)

Terufumi Kato

Employment: Eli Lilly (I)

Honoraria: Chugai Pharma, Roche, Boehringer Ingelheim, Ono Pharmaceutical, Eli Lilly, AstraZeneca, Taiho Pharmaceutical, Pfizer, Bristol-Myers Squibb Japan, Merck Sharp \& Dohme, Novartis, Sumitomo Dainippon, Quintiles, Takeda Consulting or Advisory Role: AstraZeneca, MSD, Eli Lilly, Chugai Pharma, Nitto Denko, AbbVie, Merck Serono, Pfizer

Research Funding: Chugai Pharma (Inst), Merck Sharp \& Dohme (Inst), Kyowa Hakko Kirin (Inst), Pfizer (Inst), Taiho Pharmaceutical (Inst), AstraZeneca (Inst), Eli Lilly (Inst), AbbVie (Inst), Astellas Pharma (Inst), Ono Pharmaceutical (Inst), Merck Serono (Inst), Kyorin, Regeneron

\section{Rodryg Ramlau}

Consulting or Advisory Role: BMS, Boehringer Ingelheim, MSD Oncology, Merck, Roche, Novartis, Takeda, AstraZeneca, Pfizer

Travel, Accommodations, Expenses: BMS, Boehringer Ingelheim, Roche, AstraZeneca

Silvia Novello

Speakers' Bureau: AstraZeneca, MSD, Bristol-Myers Squibb, Roche, Pfizer, Eli Lilly, Takeda, AbbVie, Boehringer Ingelheim, Bayer

\section{Sreekanth Reddy}

Employment: Inspire Sleep (I)

Honoraria: Medtronic, Celgene

Speakers' Bureau: Celgene

Hans-Georg Kopp

Honoraria: MSD Oncology, Boehringer Ingelheim, LEO Pharma, PharmaMar, Roche, Pfizer, Chugai Pharma, Takeda

Consulting or Advisory Role: MSD Oncology, Bristol-Myers Squibb, Sanofi, Roche, AstraZeneca

Travel, Accommodations, Expenses: Sanofi, Eli Lilly, Amgen, Novartis,

PharmaMar, Boehringer Ingelheim, MSD Oncology, Bristol-Myers Squibb

Bilal Piperdi

Employment: Merck Sharp \& Dohme Corp., a subsidiary of Merck \& Co., Inc., Kenilworth, NJ, USA

Stock and Other Ownership Interests: Merck Sharp \& Dohme Corp.

a subsidiary of Merck \& Co., Inc., Kenilworth, NJ, USA

\section{Xiaodong Li}

Employment: Merck Sharp \& Dohme Corp., a subsidiary of Merck \& Co., Inc., Kenilworth, NJ, USA

Stock and Other Ownership Interests: Merck Sharp \& Dohme Corp., a subsidiary of Merck \& Co., Inc., Kenilworth, NJ, USA

Travel, Accommodations, Expenses: Merck Sharp \& Dohme Corp., a subsidiary of Merck \& Co., Inc., Kenilworth, NJ, USA

Thomas Burke

Employment: Merck Sharp \& Dohme Corp., a subsidiary of Merck \& Co., Inc., Kenilworth, NJ, USA

Stock and Other Ownership Interests: Merck Sharp \& Dohme Corp.,

a subsidiary of Merck \& Co., Inc., Kenilworth, NJ, USA

\section{Luis Paz-Ares}

Leadership: Genomica, EMA SAG (I)

Honoraria: Genentech, Eli Lilly, Pfizer, Boehringer Ingelheim, Bristol-Myers Squibb, MSD, AstraZeneca, Merck Serono, PharmaMar, Novartis, Celgene, Sysmex, Amgen, Incyte, Sanofi

Research Funding: BMS, AstraZeneca

Travel, Accommodations, Expenses: Roche, AstraZeneca, MSD, Bristol-Myers Squibb, Pfizer, Takeda

Other Relationship: Novartis (I), Ipsen (I), Pfizer (I), Servier (I), Sanofi (I), Roche (I), Amgen (I), Merck (I), Roche

No other potential conflicts of interest were reported. 